\title{
How to build a nanotech company
}

\author{
What do you need to consider when launching a scientific start-up? James Vicary highlights what in his \\ views are the three main issues.
}

$\mathrm{T}$ he end of a $\mathrm{PhD}$ or postdoctoral position is a challenging time for any researcher. The pathways available seem to be either to stay in academia or to leave and probably end up working for a large company. Neither of those satisfied my aspirations as I approached the end of my postdoc in 2011. I did not want to lose the connection with science and technology, but I also needed a fresh challenge. When the idea of setting up a company came up in conversation with a couple of professors from the university, it had a certain appeal for me. Being my own boss, setting my own course and making something out of nothing seemed like the perfect way to straddle the divide between academia and industry.

I've learned a lot since those early days when my co-founders and I first set up NuNano, a company specializing in the design and manufacture of probes for atomic force microscopy (AFM). I would like to share my views on the three main issues that are worth considering when transforming a research project into a fully fledged nanotech enterprise.

\section{Covering the basics}

Setting up a scientific company has some considerable requirements, including access to laboratory space and multimillion-pound equipment. This is very different from many other start-ups - for example, a digital start-up might require only office space and a couple of computers. For NuNano, the obvious solution was to start by talking to our university. But we did not assume any right to access to their kit just because of our connections as fellows, staff or alumni of the university. Many universities are becoming wise to the opportunity to monetize their facilities by hiring them out to companies for an eyewatering amount of money. Managing our relationship with our host university has been key to our success.

The beauty of being rooted in a host university was that, through colleagues and $\mathrm{PhD}$ students, it provided NuNano with an early testbed for our AFM probes. In many instances, these testers were so happy with our product that they became early adopters and a useful source of revenue once we were in full production.

\section{Funding your enterprise}

Figuring out how to fund any enterprise can be daunting. There are lot of pockets of money out there, but we found that our approach to funding had to follow a rather piecemeal format in the early years as we got ourselves up to full production. This is completely normal.

Initial investment came from us as company founders, both in terms of hard cash and time spent working on ideas and product development. We made sure that we were business-like about these arrangements, and a memorandum of understanding ensured clarity about investment and company ownership from day one.

Later funding came from various other sources. There are several government schemes available to help start-ups, especially in terms of research and development. We had to meet the criteria of being a 'small and medium-sized enterprise' and demonstrate that the funding would lead to innovation, create and sustain jobs, and generally benefit 'UK plc'. A condition of much of the funding that we received was that we had to raise an equivalent amount of money, known as match funding, from alternative sources.

We also received grants in the form of 'add-on' funding enabling attendance at training courses and access to consultancy services, both of which we found incredibly useful as we were building our business skills and knowledge. One additional way in which we generated early revenue was by using our skill set to undertake bespoke projects and AFM imaging. A mix of agility, imagination and tenacity helped us to navigate funding our business.

\section{Product development}

A third challenge when developing an idea into a product is deciding whether to outsource the prototyping or build in-house.

Initially, we outsourced some of our prototyping to another company. I had a certain set of expectations based on what I believed their equipment would and could do. Unfortunately, these were not met, but at least I learnt a lesson about the importance of clear communication when outsourcing work.

In retrospect, it was unlikely that we were going to get exactly what we expected the first time that another company worked on the prototype. Part of the process is as much about learning what does not work as celebrating what does. Generally speaking, any company contracted to do the work is not paid to think or problem-solve these issues, just to follow the instructions they have been given.

Ultimately, we found a way to get access to the equipment that we needed to be able to do our own prototyping. The downside to this approach is that although we made enormous gains from being hands-on, including problem-solving as processing issues arose, I did have to spend a lot of time in the cleanroom. Although I really enjoy the lab work, running the company requires a considerable amount of time spent on administrative tasks. These are essential to keep running day-to-day and to survive in the long run. We are now able to employ people in the lab, but, in the beginning, we had to juggle a number of conflicting demands.

There is huge value in being an academic-entrepreneur, straddling the divide between academia and business. Many great ideas within the scientific community could bring benefits of all sorts if they were taken down a commercial route by the academics themselves.

Having navigated equipment access, funding issues and product development, we are now shipping our AFM probes out regularly to a growing base of customers. That feeling is truly exhilarating and well worth the challenges along the way.

JAMES VICARY is Managing Director of NuNano Ltd and a visiting industrial fellow at the School of Physics, University of Bristol, Bristol, UK. e-mail:james@nunano.com

\section{Competing financial interests}

J.V. is the co-founder, managing director and a shareholder of NuNano, the company referred to in this article. 